\title{
Bristol Stool Type 7
}

National Cancer Institute

\section{Source}

National Cancer Institute. Bristol Stool Type 7. NCI Thesaurus. Code C125400.

Liquid consistency with no solid pieces; indicative of diarrhea. 